\title{
Multipath Reflectivity Estimation in Urban Environments for Synthetic Aperture Radar Images
}

\author{
I. de Arriba-Ruiz, F. Pérez-Martínez \\ Departamento de Señales, Sistemas y \\ Radiocomunicaciones \\ Universidad Politécnica de Madrid \\ Avda. Complutense s/n, 28040 Madrid \\ \{imanol, felix $\} @$ gmr.ssr.upm.es
}

\author{
J.M. Muñoz-Ferreras \\ Departamento de Teoría de la Señal y \\ Comunicaciones \\ Universidad de Alcalá \\ Ctra. Madrid-Barcelona, 28805 Alcalá de Henares, Madrid \\ jm.munoz@uah.es
}

\begin{abstract}
Synthetic Aperture Radar (SAR) images a target region reflectivity function in the multi-dimensional spatial domain of range and cross-range. SAR synthesizes a large aperture radar in order to achieve a finer azimuth resolution than the one provided by any on-board real antenna. Conventional SAR techniques assume a single reflection of transmitted waveforms from targets. Nevertheless, today's new scenes force SAR systems to work in urban environments. Consequently, multiple-bounce returns are added to directscatter echoes. We refer to these as ghost images, since they obscure true target image and lead to poor resolution. By analyzing the quadratic phase error $(Q P E)$, this paper demonstrates that Earth's curvature influences the defocusing degree of multipath returns. In addition to the QPE, other parameters such as integrated sidelobe ratio (ISLR), peak sidelobe ratio $(P S L R)$, contrast $(C)$ and entropy $(E)$ provide us with the tools to identify direct-scatter echoes in images containing undesired returns coming from multipath.
\end{abstract}

Keywords - synthetic aperture radar, multipath, Earth's curvature, ghost, defocus, quadratic phase error, integrated sidelobe ratio, peak sidelobe ratio, contrast, entropy.

\section{INTRODUCTION}

Synthetic Aperture Radar (SAR) offers improvement in range resolution and, especially, in azimuth resolution [1-2] in order not only to detect, locate and identify objects, but to generate a spatial, visual representation (that is, an image) of the radar reflectivity of an illuminated scene [3].

The name of the technique alludes to the concept of signal synthesis, which involves the coherent summation of the signals produced by transmitting and receiving from a sequence of equally spaced flight path locations, which are synchronized with aircraft speed [4]. The aircraft movement is necessary to synthesize a large enough array to fulfil azimuth accuracy requirements. Reception of signals out of these ideal positions leads to errors that cause the radar coherence loss and a decline of image quality.

At the same time, a good range resolution is achieved by

This work has been supported by Project TEC2008-02148 of the Spanish National Board of Scientific and Technology Research. using pulse compression techniques. For this purpose, it is very common to use linear frequency modulated pulses (chirped pulses). That being said, unlike conventional radars, SAR systems are able to illuminate a lot of resolution cells simultaneously and to separate reflected echoes from each cell by using pulse and beam compression techniques.

The use of centimetric and millimetric wavelengths offers many advantages against optic and infrared image systems. Among other things, an all-weather, day/night capability to obtain images, which turns the SAR technique into an 'alltime' system.

The dimensions of a SAR image are range and azimuth (cross-range, which is related to the Doppler effect). This way, scatterers situated in the same range cell can be differentiated in azimuth. Range resolution $\left(\rho_{r}\right)$ improves when a larger bandwidth is transmitted $(B)$, whereas azimuth resolution $\left(\rho_{a}\right)$ is better if the change of the target aspect angle during illumination time $(\Delta \theta)$ is bigger, and it occurs as the synthetic aperture length increases [1]:

$$
\begin{aligned}
& \rho_{r}=\frac{c}{2 \cdot B}, \\
& \rho_{a}=\frac{\lambda}{2 \cdot \Delta \theta},
\end{aligned}
$$

where $c$ is the speed of light and $\lambda$ is the wavelength.

SAR radars belong to SLAR (Side-Looking Airborne Radar) family. This kind of radars work on board aircrafts and satellites, steering their antenna beam to a direction which is approximately perpendicular to flight path. As a result, an electromagnetic image of a ground swath is obtained. The SAR concept includes different imaging modes, as well as many applications both in military and civilian fields. This has turned the SAR system into a prestigious detection, location and identification technique with the passage of time.

Nevertheless, in spite of being an 'all-time' system, there are other effects that SAR techniques are not able to face up to so efficiently. They are connected with a series of internal and external factors to the SAR system -propagation and targets behaviour; transmission, reception and processing systems; 
aircraft motion errors- whose aim is to decline images, endangering their quality and their accuracy in terms of resolution. Among them, must be mentioned the well-know speckle noise, multipath effect or phase noise.

The purpose of this article is to model and simulate scenes affected by multipath, which is the consequence of propagation and targets behaviour. The exhaustive characterization of this degrading phenomenon is absolutely essential in order to be able to design effective mitigation techniques in the near future. Some innovative multipath mitigation techniques will just be mentioned without going into details.

\section{CHARACTERIZATION OF MULTIPATH IN SAR}

Multipath phenomenon is the result of the superposition of a direct echo and one or several reflected returns. In a radar system, it causes a variation of the received power from a target owing to constructive and destructive interferences that take place in reception [5-6].

Furthermore, a noisy component can be added to these echoes as a result of diffuse reflection, which is incoherent with the desired signal and it arises when a ray is incident on a rough (non-specular) surface [7-8]. This leads to an important amount of reflected rays in several spatial directions, and it can be regarded as an additive noise that is added to the system thermal noise.

Today's new scenes force SAR systems to work in urban environments (rich scattering environments). In this context, multiple-bounce returns are additionally superposed to the direct-scatter echo, producing spurious and random patterns (ghosting artifacts) around the true target in the formed SAR image. In case these additional echoes are focused and their amplitudes are significant, it can be hard to distinguish between the direct return and the ghost echoes. Therefore, as well as obscuring true target image and leading to poor resolution, they can mask the true target, making its detection almost impossible.

These reflections can take place both on points of the ground that separates the SAR system from the target and on scatterers located in the target vicinity. The analytical study of multipath varies according to scene features such as surface curvature and roughness, and results will be different in each case. For this reason, we need to differentiate between specular and diffuse multipath for curved and flat Earth.

\section{A. Specular multipath}

Multipath is specular when the surface is smooth enough. The number of reflected echoes that are received in the radar is finite. This allows us to analyze multipath with a ray model.

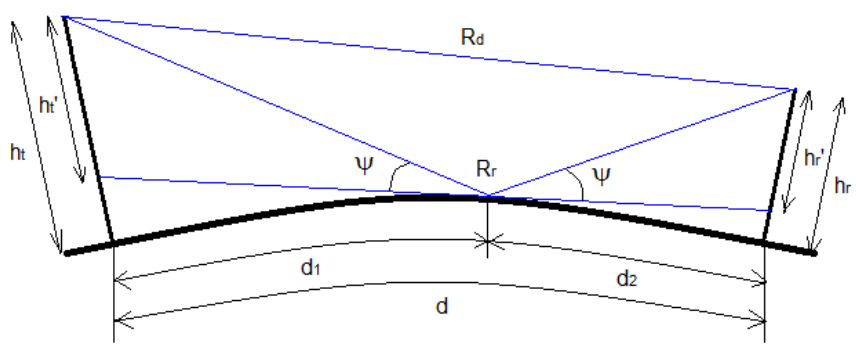

Figure 1. Multipath geometry for curved Earth [5]

It is really important to emphasize that there is only a point of Earth's surface -located on a point of the straight line that links the SAR system to the desired target- which verifies Snell's law -the incidence angle of the multipath echo on Earth's surface is the same as its reflection angle-. That is the reason why we are only considering one reflected ray, that is, a two-ray model. Other reflected rays could arise between the SAR system and the target. They would also verify Snell's law; nevertheless, they would not be incident on the target.

Fig. 1 shows multipath geometry for curved Earth [5]. It is valid for each of the azimuth positions that will be reached by the on-board SAR during its flight. The modelling of multipath is just a geometric problem: if SAR and target heights $\left(h_{t}\right.$ and $\left.h_{r}\right)$ are known, as well as the distance between them $(d)$, it is possible to work out the distance that is covered by the direct return $\left(R_{d}\right)$ and by the reflected echo $\left(R_{r}\right)$. At this point, it is straightforward to calculate their delays. The rest of parameters of Fig. 1 are the grazing angle $(\psi)$, the distance between the SAR system and the reflection point $\left(d_{1}\right)$, the distance between the target and the reflection point $\left(d_{2}\right)$, the equivalent height of the aircraft $\left(h_{t}{ }^{\prime}\right)$ and the equivalent height of the target $\left(h_{r}{ }^{\prime}\right)$.

Naturally, the reflected echo is the one that suffers more attenuation, not just because it covers a larger distance composed by two sections: the incidence on the surface and the subsequent reflection- but because it also loses part of its power when being incident on Earth's surface. Simultaneously, its phase is different from the one that the direct echo has. This causes constructive and destructive interferences in reception depending on whether the reflected ray is in phase or out of phase.

If inherent features of the medium (dry ground, wet ground, sea, etc) and SAR parameters (frequency, polarization, etc) are known, the effective specular reflection coefficient $\left(\Gamma_{s}\right)$ can be worked out [5-6, 8-9]. It shows the amount of incident ray that is reflected from a surface and it is made up of three distinct factors: the flat Earth reflection coefficient $\left(\Gamma_{h, v, c}\right)$, a factor owing to Earth's curvature divergence $(D)$ and a factor due to ground roughness $\left(\rho_{s}\right)$ :

$$
\Gamma_{s}=\Gamma_{h, v, c} \cdot D \cdot \rho_{s}
$$

As far as the flat Earth reflection coefficient is concerned, it varies according to surface electric properties, the frequency, 
the grazing angle and the signal polarization (v: vertical, h: horizontal, c: circular) in this way:

$$
\begin{gathered}
\Gamma_{v}=\frac{\varepsilon \cdot \sin (\psi)-\sqrt{\varepsilon-\cos ^{2}(\psi)}}{\varepsilon \cdot \sin (\psi)+\sqrt{\varepsilon-\cos ^{2}(\psi)}}, \\
\Gamma_{h}=\frac{\sin (\psi)-\sqrt{\varepsilon-\cos ^{2}(\psi)}}{\sin (\psi)+\sqrt{\varepsilon-\cos ^{2}(\psi)}}, \\
\Gamma_{c}=\frac{\Gamma_{h}+\Gamma_{v}}{2},
\end{gathered}
$$

where the permittivity consists of the relative permittivity $\left(\varepsilon_{r}\right)$ [10], the surface conductivity $(\sigma)[10]$ and the wavelength $(\lambda)$ :

$$
\varepsilon=\varepsilon_{r}-j \cdot 60 \cdot \sigma \cdot \lambda .
$$

As for Earth's curvature divergence factor, it takes into account the energy dispersion of the reflected echo caused by a non-flat incidence surface. Its value is approximately the unit, with the exception of the cases in which the grazing angle is very low (by high distances between radar and target) since it tends to zero:

$$
D=\frac{1}{\sqrt{1+\frac{2 \cdot d_{1} \cdot d_{2}}{r_{e} \cdot d \cdot \sin (\psi)}}},
$$

where $r_{e}$ is Earth's effective radius and is given by $4 / 3$ times Earth's radius.

Finally, the ground roughness factor takes into account the reflected echo attenuation due to the fact that the incidence surface is not completely smooth. It decreases as grazing angle increases (Fig. 2):

$$
\rho_{s}=e^{-2 \cdot\left(\frac{2 \pi \cdot \sigma_{h} \cdot \sin (\psi)}{\lambda}\right)^{2}}
$$

Flat Earth multipath involves a simplification with regard to the previous case since we are not considering Earth's inherent curvature. It facilitates calculus both of the distance covered by each ray and of the effective specular reflection coefficient, where Earth's curvature divergence factor does not have to be considered:

$$
\Gamma_{s}=\Gamma_{h, v, c} \cdot \rho_{s} .
$$

The main difference between both cases lies in results, as it will be shown in section III.

\section{B. Diffuse multipath}

The incidence of the signal on a rough surface scatters its energy towards many spatial directions, which generates multiple incoherent reflections that will reach the SAR receiver [7-8]. The problem is solved in the same way as in the previous cases. The difference lies in the fact that it is necessary to add an incoherent Gaussian noise to the received signal, whose variance is given by the product of the received power from the desired target and the square of the effective diffuse reflection coefficient $\left(\Gamma_{d}\right)$.

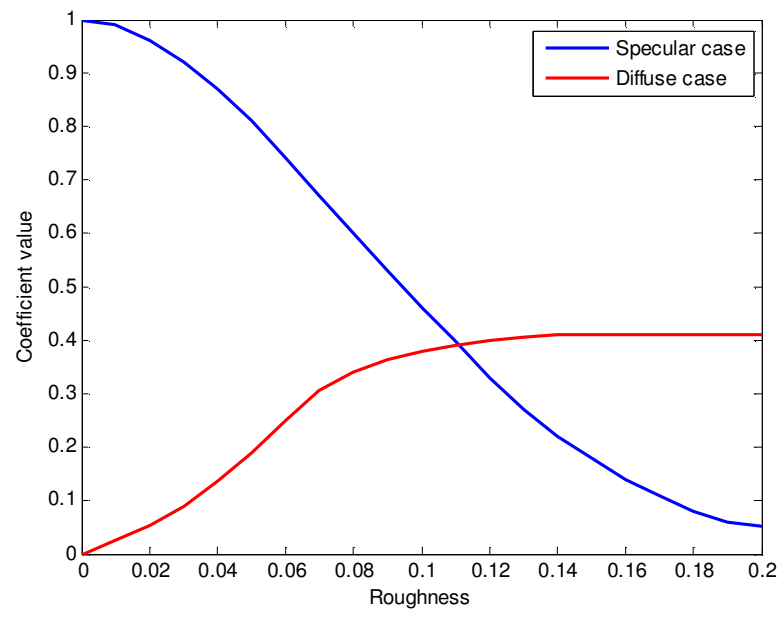

Figure 2. Evolution of $\rho_{s}$ and $\rho_{d}$ according to ground roughness [7]

The calculus of this new coefficient is almost similar to the preceding case, differing only in the ground roughness factor $\left(\rho_{s}\right)$, which is replaced by the diffuse reflection roughness coefficient $\left(\rho_{d}\right)$. This term considers a ground roughness much bigger than in the specular case. Furthermore, $\rho_{d}$ is given by an experimental curve which takes into account Earth's curvature. For this reason, it is not necessary to include Earth's curvature divergence factor in $\Gamma_{d}$ :

$$
\Gamma_{d}=\Gamma_{h, v, c} \cdot \rho_{d} .
$$

In view of $\Gamma_{d}$ dependence on grazing angle, the noise will have a higher influence on the signal when the distance between radar and target is small (by high grazing angles). Fig. 2 shows the evolution of both $\rho_{s}$ and $\rho_{d}$ according to ground roughness, which is given by the following expression:

$$
\text { Roughness }=\frac{\sigma_{h}}{\lambda} \cdot \sin (\psi),
$$

where $\sigma_{h}$ is the standard deviation of ground height.

\section{Multipath mitigation techniques}

Nowadays, there are several techniques aimed at multipath reduction in SAR, highlighting among them the most innovative ones such as Time Reversal SAR [11] and IRAMS (Image Reconstruction Algorithm for Multipath Scattering) algorithm [12].

\section{RESULTS}

In order to form SAR images considering a scene with specular multipath (it is straightforward to extend it to a situation of diffuse multipath), one of the most efficient SAR image formation algorithms has been used -RMA, Range Migration Algorithm-. Its ability to compensate range curvature accurately must be highlighted. 
Multipath phenomenon can be very pernicious since 'ghost echoes' can mask the true target. Nonetheless, the existence of any feature which can let us distinguish direct-scatter return from ghost echoes will sort out the problem. Two important aspects to bear in mind are the amplitude of multipath echoes and, especially, its degree of defocus in the image. The latter is given by Quadratic Phase Error $(Q P E)$, which is calculated comparing the phase history of the multipath echo scatterer $\left(R_{r}\right)$ with the phase history of an ideal scatterer situated at a distance equal to the one that is covered by the multipath ray at the central point of the synthetic aperture $\left(R_{d}\right)$ :

$$
Q P E=\frac{4 \pi}{\lambda} \cdot\left(R_{d}-R_{r}\right) .
$$

$Q P E$ widens SAR impulse response in azimuth direction; therefore, it is interesting to measure some parameters such as ISLR (Integrated Sidelobe Ratio, $\mathrm{dB}$ ) and PSLR (Peak Sidelobe Ratio, $\mathrm{dB}$ ) in this direction:

$$
\begin{gathered}
I S L R=\frac{\text { Sidelobes energy }}{\text { Mainlobe energy }} \\
P S L R=\frac{\text { Peak sidelobe intensity }}{\text { Peak mainlobe intensity }} .
\end{gathered}
$$

Both of them tend to make worse with the presence of $Q P E$. At the same time, another way to quantify the degree of defocus is by using Entropy $(E)$ and Contrast $(C)$ [13-14]. An increase of the defocusing degree will be detected when $E$ is higher and $C$ is lower:

$$
\begin{gathered}
E=-\sum_{i} \sum_{j} \bar{I}_{i, j} \cdot \ln \bar{I}_{i, j}, \\
C=\frac{\sqrt{A\left\{\left[\left|I_{i, j}\right|^{2}-A\left(\left|I_{i, j}\right|^{2}\right)\right]^{2}\right\}}}{A\left(\left|I_{i, j}\right|^{2}\right)},
\end{gathered}
$$

where $I_{i, j}$ is SAR image, $i$ and $j$ are spatial coordinates, $A(\cdot)$ operator represents the spatial mean and $\bar{I}_{i, j}$ is the normalized SAR image, given by the expression:

$$
\bar{I}_{i, j}=\frac{\left|I_{i, j}\right|^{2}}{\sum_{i} \sum_{j}\left|I_{i, j}\right|^{2}} .
$$

The most relevant simulation parameters of the used radar are: work frequency $(242.2 \mathrm{MHz})$, transmitted bandwidth (133.5 MHz), pulse repetition frequency $(320 \mathrm{~Hz})$, sampling rate $(192 \mathrm{MHz})$ and pulse length $(4 \mu \mathrm{s})$.

\section{A. Flat Earth multipath}

In this section, a case of reflection from a point of the ground that separates the SAR system from the target is addressed. Furthermore, the reflected echo covers, in its return path, the same one as it covered until reaching the target in its outward path $\left(R_{r}\right)$. The result for a punctual target is shown in Fig. 3, where we can appreciate the desired target as well as a false target on its right in consequence of multipath. Fig. 4 displays the QPE of the multipath return.

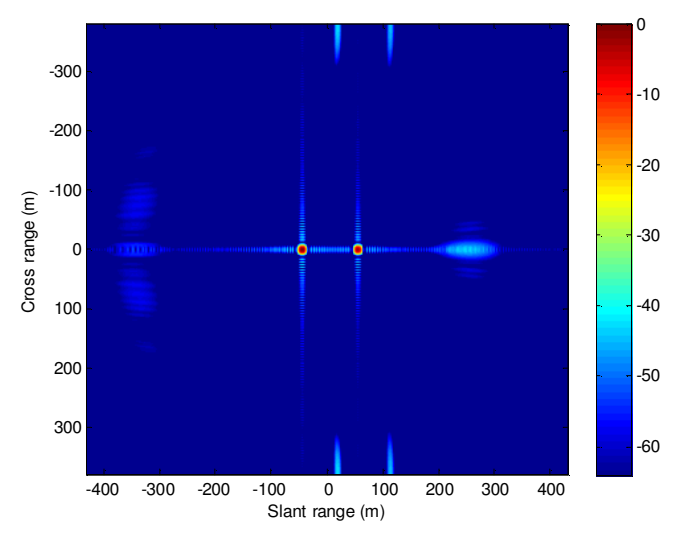

Figure 3. SAR image with multipath for flat Earth

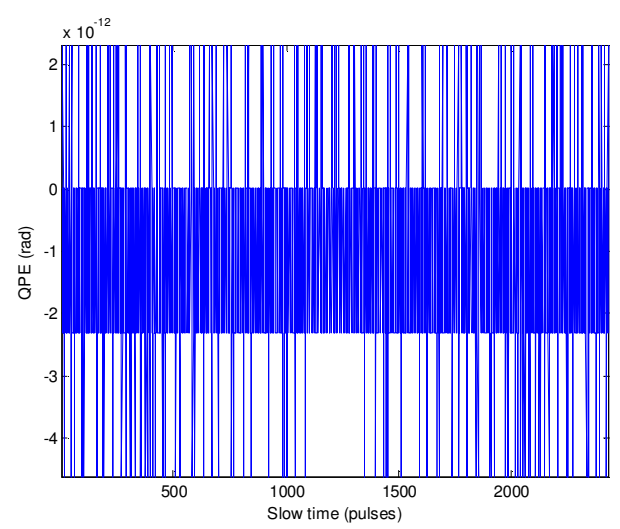

Figure 4. QPE of the multipath echo for flat Earth

The most noteworthy fact is that the multipath echo is completely focused in the image ( $Q P E$ is approximately zero). For this reason, when it comes to distinguish the true target from the undesired return coming from multipath, many doubts may arise. In geometric terms, we can state that the origin of multipath is a known point. In order to corroborate this idea, Table I demonstrates that ISLR and PSLR are very alike for both echoes.

TABLE I. PARAMETERS OF THE SAR IMAGE WITH MULTIPATH FOR FLAT EARTH

\begin{tabular}{|c|c|c|c|c|}
\hline & $I S L R$ & $P S L R$ & $E$ & $C$ \\
\hline Direct echo & -34.3800 & -42.6200 & \multirow{2}{*}{5.7783} & \multirow{2}{*}{66.8756} \\
\hline Multi. echo & -34.3761 & -42.6193 & & \\
\hline
\end{tabular}

\section{B. Curved Earth multipath}

In this case, the previous study is repeated but now Earth's curvature is taken into account. The resulting SAR image is very similar to the previous case image, so it will not be shown. The main difference is that the multipath echo is not focused in the image, in other words, a small QPE appears due to the non-existence of an origin point of multipath (Fig. 5). Therefore, it has been demonstrated that Earth's curvature influences the defocusing degree of multipath returns. 


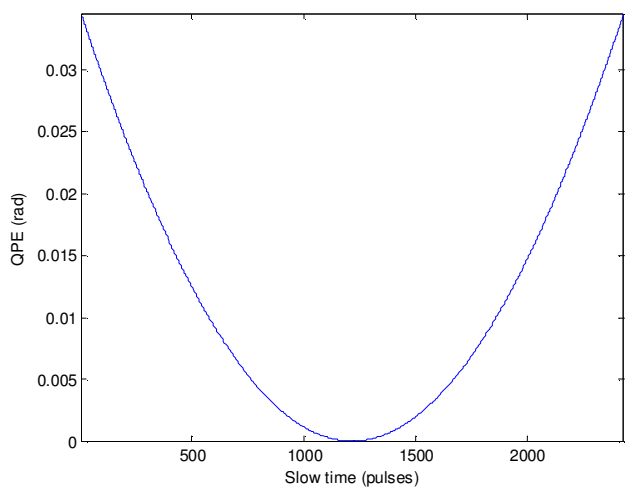

Figure 5. QPE of the multipath echo for curved Earth

Comparing the results (Table II) with the flat Earth case (Table I), we can confirm that they are consistent since the reflected echo PSLR gets worse. This parameter increases as mainlobe level is lower and sidelobes level is higher. At the same time, $E$ and $C$ also get worse -the entropy increases and the contrast decreases, that is, the defocusing is higher-.

TABLE II. PARAMETERS OF THE SAR IMAGE WITH MULTIPATH POR CUR VED EARTH

\begin{tabular}{|c|c|c|c|c|}
\hline & $I S L R$ & PSLR & $E$ & $C$ \\
\hline Direct echo & -34.3799 & -42.6200 & \multirow{2}{*}{5.7796} & \multirow{2}{*}{66.8526} \\
\hline Multi. echo & -34.3763 & -42.6183 & & \\
\hline
\end{tabular}

\section{Other analyzed scenes}

Another case of flat Earth is analyzed below in which the reflected echo covers, in its return path, the same one as the direct echo does $\left(R_{d}\right)$. The obtained SAR image is shown in Fig. 6:

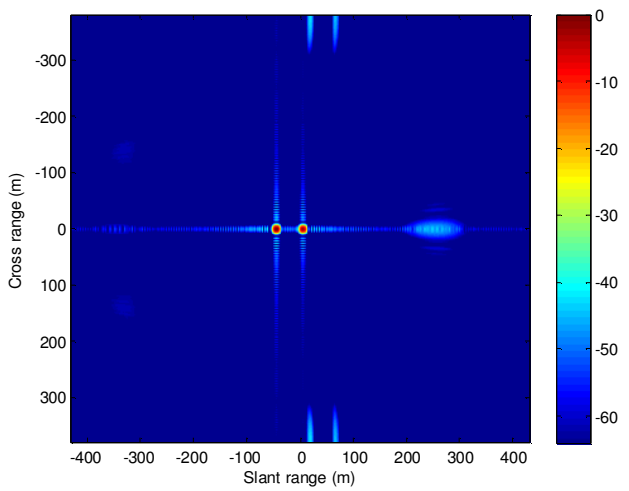

Figure 6. SAR image with multipath for flat Earth (example 2)

Again, the $Q P E$ of the multipath echo is presented (Fig. 7) as well as the value of the main parameters of the obtained SAR image (Table III).

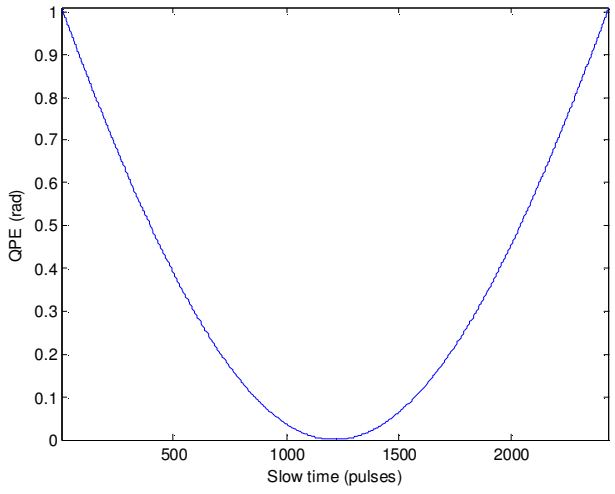

Figure 7. QPE of the multipath echo for flat Earth (example 2)

TABLE III. PARAMETERS OF THE SAR IMAGE WITH MULTIPATH FOR FLAT EARTH (EXAMPLE 2)

\begin{tabular}{|c|c|c|c|c|}
\hline & $I S L R$ & PSLR & $E$ & $C$ \\
\hline Direct echo & -34.3798 & -42.6197 & \multirow{2}{*}{5.7712} & \multirow{2}{*}{67.1710} \\
\hline Multi. echo & -34.3679 & -42.6187 & & \\
\hline
\end{tabular}

The multipath echo is considerably more defocused than in the previously proposed geometry. The proof is that the $Q P E$ is quite higher. Despite being a case of flat Earth, the cause is again the non-existence of an origin point of multipath -only the scene of flat Earth explained previously implies no $Q P E-$. For curved Earth, the result is very similar but with a defocusing slightly higher $-C$ gets worse-. Once more we can check that the defocusing degree of multipath returns is higher for curved Earth.

By simulating other multipath scenes of interest, as an urban environment in which rays can be reflected from target surrounding scatterers more than once, we have verified that the defocusing degree of multipath returns is considerable. This way, it would be easier to distinguish the direct-scatter return from among ghost echoes. In the following example, the multipath return, after being reflected from the target, reaches a scatterer close to the target which retroscatters the signal. A part of it is again incident on the desired target, which reflects the signal towards the SAR system. Fig. 8 displays the resulting SAR image:

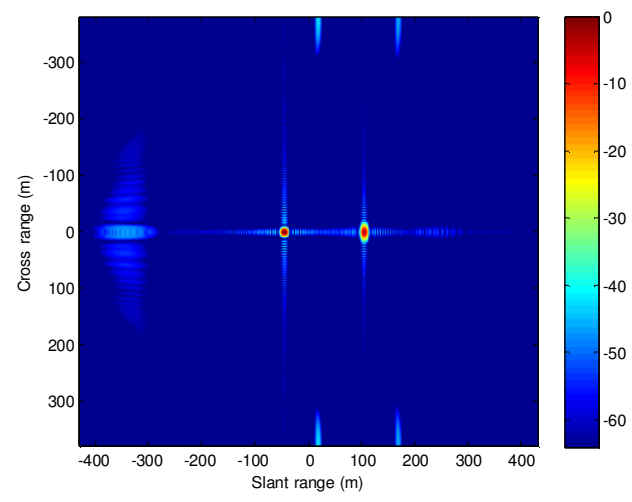

Figure 8. SAR image in an urban environment 
As for the $Q P E$ of the multipath return (Fig. 9), it is very high at the extremes of the synthesized aperture.

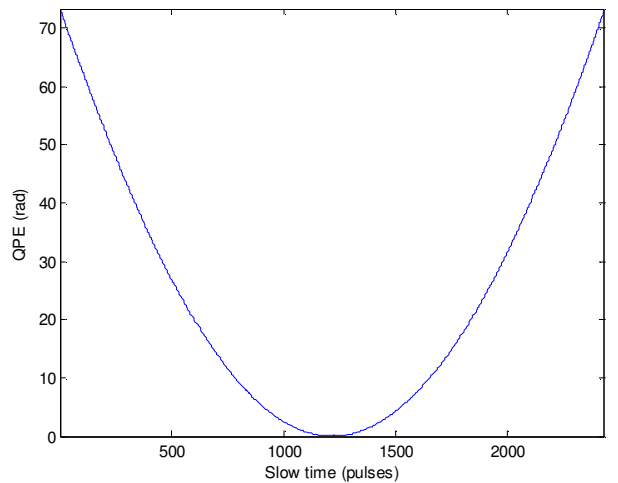

Figure 9. QPE of the multipath echo in an urban environment

Due to the presence of a so high $Q P E$, all the parameters of the SAR image get considerably worse (Table IV) $-I S L R$ and $P S L R$ are higher in the multipath echo; the entropy increases and the contrast decreases with regard to the previous cases-. It will facilitate the detection of the true target.

TABLE IV. PARAMETERS OF THE SAR IMAGE IN AN URBAN ENVIRONMENT

\begin{tabular}{|c|c|c|c|c|}
\hline & $I S L R$ & PSLR & $E$ & $C$ \\
\hline Direct echo & -34.3661 & -42.5992 & \multirow{2}{*}{6.0427} & \multirow{2}{*}{59.0332} \\
\hline Multi. echo & -17.4549 & -41.1462 & & \\
\hline
\end{tabular}

\section{CONCLUSIONS}

In this article, we have shown the influence of Earth's curvature on the defocusing degree of multipath returns. In the first case, as far as flat Earth is concerned, the origin of multipath is a known point, and for this reason, there is no $Q P E$ in the SAR image. As for curved Earth, that origin point does not exist with the result that a $Q P E$ appears. It is higher with an increase of the altitude difference between the onboard SAR system and the target, of the synthetic aperture length and of the frequency. Nevertheless, it will not involve an excessive defocusing in any case; therefore, this kind of multipath can be really harmful since it can lead to a ghost target very similar and very close to the true target. The rest of infinite possible cases of multipath appear considerably more defocused, so their detection is much easier (for instance, in urban environments).

\section{REFERENCES}

[1] W.G. Carrara, R.S. Goodman, R.M. Majewski, Spotlight Synthetic Aperture. Signal Processing Algorithms, Artech House, 1995.

[2] C.A. Wiley, Synthetic Aperture Radars, IEEE Trans. on Aerospace and Electronic Systems, Vol. AES-21, No. 3, pp. 440-443, May 1995.

[3] D.L. Mensa, High Resolution Radar Imaging, Norwood, MA, Artech House, 1981.

[4] I.G. Cumming, F.H. Wong, Digital Processing of Synthetic Aperture Radar Data. Algorithms and Implementation, Artech House, 2005.

[5] J.M. Hernando-Rábanos, Transmisión por Radio, Editorial Centro de Estudios Ramón Areces, S.A. Madrid, 1998.
[6] B.R. Mahafza, Introduction to radar analysis, CRC Press, 1998.

[7] D.K. Barton, Multipath Fluctuation Effects in Track-While-Scan Radar, IEEE Transactions on Aerospace and Electronic Systems, Vol. AES-15, Nov. 1979.

[8] E. Daeipour, W.D. Blair, Y. Bar-Shalom, Bias Compensation and Tracking with Monopulse Radars in the Presence of Multipath, IEEE Transactions on Aerospace and Electronic Systems, Vol. 33, No. 3, July 1997.

[9] J.G. Teti Jr., Wide-Band Airborne Radar Operating Considerations for Low-Altitude Surveillance in the Presence of Specular Multipath, IEEE Transactions on Antennas and Propagation, Vol. 48, No. 2, pp. 176-191, February 2000.

[10] ITU-R P.527 Recommendation, International Telecommunication Union - Radiocommunication Sector.

[11] Y. Jin, J.M.F. Moura, M. Mulford, N. O'Donoughue, A. Samuel, Time Reversal Synthetic Aperture Radar Imaging in Multipath, Proc. 41st Annual Asilomar Conference on Signals, Systems, and Computers (Asilomar '07), pp.1812-1816, Pacific Grove, CA, November 2007.

[12] D.A. Garren, J.S. Goldsteinl, D.R. Obuchon, R.R. Greene, J.A. North, SAR Image Formation Algorithm with Multipath Reflectivity Estimation, Radar Conference, 2004.

[13] L. Xi, L. Guosui, J. Ni, Autofocusing of ISAR Images based on Entropy Minimization, IEEE Transactions on Aerospace and Electronic Systems, Vol. 35, No. 4, pp. 1240-1252, Octubre 1999.

[14] F. Berizzi, G. Corsini, Autofocusing of Inverse Synthetic Aperture Radar Images using Contrast Maximization, IEEE Transactions on Aerospace and Electronic Systems, Vol. 32, No. 3, pp. 1185-1191, Julio 1996. 